\title{
Efecto del Almacenamiento a Diferentes Temperaturas sobre la Calidad de Tuna Roja (Opuntia ficus indica (L.) Miller)
}

\author{
Carlos E. Ochoa ${ }^{(1)}$ y José A. Guerrero ${ }^{(2)^{*}}$
}

(1) Universidad de las Américas, Puebla, Programa de Doctorado en Ciencia de Alimentos. Sta. Catarina Mártir. C.P.72810, Cholula, Puebla-México (e-mail: carlosenriqueov@hotmail.com)

(2) Depto. de Ingeniería Química, Alimentos y Ambiental, Universidad de las Américas Puebla,

Sta. Catarina Mártir. C.P.72810, Cholula, Puebla-México (e-mail: angel.guerrero@udlap.mx)

* autor a quien debe ser dirigida la correspondencia

Recibido Abr. 18, 2011; Aceptado Jun. 13, 2011; Versión Final recibida Jul. 27, 2011

\section{Resumen}

Se estudia el efecto del almacenamiento a diferentes temperaturas sobre la calidad de tuna roja (Opuntia ficus indica (L.) Miller), variedad San Martín. El fruto se almacenó a $4 \pm 1,9 \pm 2$ y $28 \pm 2^{\circ} \mathrm{C}$ para determinar su vida útil. Se realizó semanalmente la caracterización fisicoquímica, enzimática, antioxidante y microbiológica durante el almacenamiento, hasta observar características no aptas para el consumo. Se observó que el tiempo y la temperatura de almacenamiento son factores que afectan de manera significativa $(P<0,05)$ a la pérdida de peso, la textura y la actividad de la polifenoloxidasa en cáscara de tuna. El contenido de compuestos fenólicos, la capacidad antioxidante y la actividad de la enzima pectinesterasa en pulpa de tuna no presentaron diferencia significativa $(P>0,05)$ a las diferentes temperaturas de almacenamiento. Sin embargo, la actividad antioxidante presentó un aumento significativo con el tiempo.

Palabras clave: tuna roja, opuntia, almacenamiento, vida útil, capacidad antioxidante

\section{Effect of the Storage at Different Temperatures on the Quality of Red Prickle Pear (Opuntia ficus indica (L.) Miller)}

\begin{abstract}
The effect of the storage at different temperatures on the quality of red prickle pear (Opuntia ficus indica (L.) Miller) San Martín variety. The fruit was stored at $4 \pm 1,9 \pm 2$, and $28 \pm 2^{\circ} \mathrm{C}$ for determining its shelf life. Once a week, physicochemical, enzymatic, antioxidant and microbiological characteristics were evaluated until fruit showed no edible characteristics. Time and storage temperature were the two parameters that significantly affected $(p<0.05)$ weight loss, texture and activity of polyphenoloxidase in pear peel. Phenolic compounds, antioxidant capacity, and pectinesterase activity did not show significant difference $(p>0.05)$ during storage time. However, antioxidant capacity presented a significant increase with time.
\end{abstract}

Keywords: red prickly pear, opuntia, storage, shelf life, antioxidant capacity 


\section{INTRODUCCIÓN}

La tuna (Opuntia spp.) es una fruta perteneciente a la familia de las cactáceas y ha sido hasta ahora la más explotada y comercializada en México. Es muy apreciada por su sabor característico y por los múltiples atributos que posee (Ochoa y Guerrero, 2010). Es un fruto que se consume en fresco y recientes estudios han demostrado que la tuna contiene altos niveles de agentes antioxidantes tales como ácido ascórbico, compuestos fenólicos y pigmentos betalaínicos (Tesoriere et al. 2005); así como altas concentraciones de compuestos como calcio, magnesio, prolina y taurina, que le confieren características de un alimento funcional (Galati et al. 2003). Para la distribución y comercialización se necesita una fruta de buena presentación y calidad; lo cual no se logra de manera satisfactoria ya que el principal inconveniente de la tuna es la corta estacionalidad de su producción (Ochoa y Guerrero, 2010). Además, la tuna es un fruto perecedero que se abarata en las épocas de mayor producción (Esquivel, 2004); por tanto, es una realidad la necesidad de buscar y probar tecnologías satisfactorias de conservación, así como técnicas de manejo poscosecha, empaque y embalaje, que permitan un mejor aprovechamiento de la tuna.

Actualmente se ha buscado la combinación de tecnologías alternativas y/o tradicionales para la conservación de alimentos. El almacenamiento a bajas temperaturas es el método de conservación más utilizado para productos en fresco. Este ayuda a mantener una adecuada apariencia, reducir la respiración y reacciones enzimáticas, así como disminuir el ablandamiento y crecimiento microbiano (Aguayo, 2003). Por otro lado, el almacenamiento bajo atmósferas modificadas ha probado ser una tecnología adecuada para alargar la vida útil de frutas y hortalizas (Kader et al. 1989). El objetivo de este estudio fue investigar el efecto de la temperatura de almacenamiento y humedad relativa sobre las características fisicoquímicas, enzimáticas, antioxidantes y microbiológicas de tuna roja variedad San Martín.

\section{MATERIALES Y MÉTODOS}

\section{Fruta}

Se utilizó tuna roja variedad San Martín (Opuntia ficus indica (L.) Miller) cosechada en la comunidad de San Sebastián Villanueva, Puebla. La tuna fue cosechada manualmente por la mañana un día antes del inicio del almacenamiento. Se seleccionaron aquellas tunas con una coloración roja uniforme, sin daños físicos y se higienizaron con una solución de cloro (200 ppm) durante 1 min justo antes de inicio del almacenamiento. Las tunas fueron divididas en 3 lotes y empacadas en cajas plásticas de polietileno cristal ( 3 tunas por caja $=299,0 \pm 22,6 \mathrm{~g}$ ) de $15 \times 15 \times 10 \mathrm{~cm}$ y se almacenaron a temperaturas de $4 \pm 1,9 \pm 2$ y $28 \pm 2^{\circ} \mathrm{C}$ y humedades relativas de $90 \pm 4 \%, 85 \pm 5 \%$ y de $75 \pm 5 \%$, respectivamente. Para cada temperatura se almacenaron tres réplicas. La tuna entera se analizó en base a pérdida de peso, textura (penetración), color y carga microbiana superficial. La tuna pelada se analizó en base a color y textura (penetración).

\section{Jugo para análisis}

La tuna fue lavada, secada y pelada. Varias tunas peladas fueron trituradas usando un multiprocesador de alimentos doméstico a baja velocidad. Las semillas fueron separadas usando tela de manta de cielo (para quesería) y el jugo fue usado inmediatamente para el análisis fisicoquímico $(\mathrm{pH}$, sólidos solubles totales y acidez titulable), enzimático (pectinesterasa y polifenoloxidasa), antioxidante (actividad antioxidante y compuestos fenólicos). Todos los análisis se realizaron por triplicado.

$\mathrm{pH}$

Se determinó siguiendo el método 981.12 de la AOAC (2000) que consta de la inmersión directa del electrodo en el jugo de tuna utilizando un potenciómetro ORION modelo 420 A (MA, EUA).

\section{Sólidos solubles totales}

Se determinaron siguiendo el método 932.12 de la AOAC (2000) utilizando un refractómetro digital ATAGO 0-45 (Tokio, Japón). 


\section{Acidez titulable}

Se determinó por el método 942.15 de la AOAC (2000), utilizando una solución valorada de $\mathrm{NaOH}$ $0,1 \mathrm{~N}$, y fenolftaleína como indicador. Se informa el porcentaje como ácido cítrico.

Índice de madurez

Se expresó como el cociente de sólidos solubles totales (\% $\mathrm{p} / \mathrm{p})$ y acidez titulable de la fruta $(\%$ $\mathrm{p} / \mathrm{p})$.

\section{Pérdida de peso}

Se evaluó en tuna entera, pulpa y cáscara utilizando el método de la AOAC (2000). El porcentaje de pérdida de peso se calcula en base a la diferencia de peso entre el peso inicial y el final usando una balanza Scout ${ }^{\mathrm{TM}}$ Pro (Ohaus Co., Zurich, Suiza) de $2000 \pm 0,1 \mathrm{~g}$.

\section{Color}

Se evaluaron los parámetros $L$ (luminosidad), $a$ (+ rojo, - verde) y $b$ (+ amarillo, - azul), de la escala de Hunter, utilizando un colorímetro COLORGARD System 05 (Virginia, EUA).

\section{Penetración}

Se determinó el esfuerzo necesario para penetrar la pulpa y cáscara utilizando un texturómetro TA- XT2 Stable Micro Systems (Haslemere, Inglaterra). Se siguió el método reportado por Del Valle et al. (2005), utilizando una aguja de $3 \mathrm{~mm}$ de diámetro, distancia de penetración de $5 \mathrm{~mm}$ en pulpa y $2,5 \mathrm{~mm}$ en cáscara y a una velocidad de $1 \mathrm{~mm} / \mathrm{s}$.

\section{Pectinesterasa (PE)}

Se realizó de acuerdo a la técnica propuesta por Rouse et al. (1954) con modificaciones. Se colocaron $10 \mathrm{~mL}$ de una solución de pectina cítrica al $1 \%$, en un vaso de doble pared con agua circulando a $30^{\circ} \mathrm{C}$. Posteriormente, se adicionaron $10 \mathrm{~mL}$ de jugo de tuna seguido de $0,1755 \mathrm{~g}$ de cloruro de sodio. Se adicionó $\mathrm{NaOH} 2$ y $0,5 \mathrm{~N}$ para alcanzar el pH inicial de 7,5. Una vez alcanzado el pH se inició el registro del tiempo y cada dos minutos se le agregaron gotas de $\mathrm{NaOH} 0,02 \mathrm{~N}$ para aumentar el $\mathrm{pH}$ a 7,5 durante 10 minutos.

$$
\mathrm{UAE}=\frac{\mathrm{m}^{*} \mathrm{~N}_{\mathrm{NaOH}}}{\mathrm{V}_{\text {jugo }}}
$$

\section{Polifenoloxidasa (PPO)}

Se realizó en pulpa y cáscara siguiendo la técnica propuesta por Pizzocaro et al. (1993) con modificaciones. Se tomaron $5 \mathrm{~g}$ de pulpa o cáscara de tuna y se mezclaron con $5 \mathrm{~mL}$ de buffer Mcllvaine pH 6,5. La mezcla se centrifugó a $4000 \mathrm{rpm}$ durante 40 minutos a $4^{\circ} \mathrm{C}$. En una celda de cuarzo, se mezclaron $0,5 \mathrm{~mL}$ de extracto enzimático con $1 \mathrm{~mL}$ de solución de catecol $(0,175 \mathrm{M})$ y $2 \mathrm{~mL}$ de buffer citrato-fosfato (Mcllvaine). Se analizó la actividad de la polifenoloxidasa a $420 \mathrm{~nm}$ y $25^{\circ} \mathrm{C}$, midiendo la absorbacia durante 3 minutos cada 10 segundos. La absorbancia se midió usando un espectrofotómetro UVIVIS marca UNICO ${ }^{\circledR}$ modelo $2800 \mathrm{H}$ (Dayton, NJ, EUA). Se realizó una gráfica con los datos (absorbancia con respecto al tiempo). La porción lineal de la gráfica se utilizó para determinar la actividad de la polifenoloxidasa. Una unidad de actividad enzimática $(U A E)$ se define como la cantidad de enzima que se requiere para dar un cambio de absorbancia de 0,001/min $\mathrm{mL}$ (o g).

$$
U A E=\frac{m^{*} 60 * V}{v^{*} 0.001^{*} g}
$$

\section{Actividad antioxidante (ABTS)}

La actividad antioxidante se determinó por el método ABTS siguiendo la metodología propuesta por $\mathrm{Re}$ et al. (1999) y modificada por Kuskoski et al. (2004). Se tiene que formar el radical ABTS ${ }^{+}$ colocando $0,0033 \mathrm{~g}$ de persulfato de potasio y $0,0194 \mathrm{~g}$ del reactivo ABTS (2,2'-azinobis (3- 
ethylbenzthiozoline) - ácido 6-sulfónico) en un frasco de vidrio ámbar. Se añaden $5 \mathrm{~mL}$ de agua destilada. La mezcla se agita perfectamente y se deja reposar por 16 horas en la oscuridad a temperatura ambiente para utilizarse en un intervalo de 24 horas posteriores. Se realiza una mezcla etanol absoluto con el radical $\mathrm{ABTS}^{+}$hasta alcanzar una absorbancia de $0,70 \pm 0,02$ a 754 $\mathrm{nm}$. Se mezclan $3920 \mu \mathrm{L}$ de la solución de radical ABTS:etanol y se registra su absorbancia como la absorbancia inicial. Se adicionan $80 \mu \mathrm{L}$ de la muestra (jugo) y en ese momento se empieza a contar el tiempo de la reacción (tiempo cero). Concluida la reacción (7 minutos después) se registra nuevamente la lectura de la absorbancia y se considera como la absorbancia final. Con los datos anteriores se calcula el porcentaje de inhibición aplicando la siguiente fórmula:

$$
U I=\frac{A_{i}-A_{f}}{A_{i}} * 100
$$

Con el porcentaje de inhibición y la ecuación de la curva estándar del antioxidante de referencia se podrá calcular la concentración de dicho antioxidante en la muestra. La curva estándar se obtiene tomando alícuotas de una solución madre de Trolox $(0,2 \mathrm{mg} / \mathrm{mL})$. Se realizan diluciones de esta solución madre de la siguiente manera ( $\mathrm{mL}$ solución madre de Trolox:agua destilada): 0:10, 0,625:9,375, 1,25:8,75, 1,875:0,875, 2,5:7,5, 3,125:6,875, 6,25:3,75, 7,5:2,5, 8,75:1,25,10:0, para obtener 10 puntos de la curva estándar. La absorbancia se mide usando un espectrofotómetro UV/VIS marca UNICO ${ }^{\circledR}$ modelo $2800 \mathrm{H}$ (Dayton, NJ, EUA).

\section{Compuestos fenólicos}

Se determinaron por el método propuesto por Gao et al. (2000) con modificaciones. Se utiliza el reactivo de Folin-Ciocalteau usando ácido gálico como estándar. En tubo ámbar se mezclan $2 \mathrm{~mL}$ de agua destilada con $200 \mu \mathrm{L}$ de reactivo de Folin-Ciocalteau y se añaden $100 \mu \mathrm{L}$ de jugo. Esta mezcla se incuba durante 3 minutos a temperatura ambiente $\left(25^{\circ} \mathrm{C}\right)$. Se debe preparar un blanco en el cual se sustituya la cantidad de muestra por agua destilada. Se añade $1 \mathrm{~mL}$ de solución de $\mathrm{Na}_{2} \mathrm{CO}_{3}$ al $20 \%$, se mezclan perfectamente y se incuba durante 1 hora en la oscuridad a temperatura ambiente. Se lee la absorbancia a $765 \mathrm{~nm}$ con la ayuda de un espectrofotómetro UVIVIS marca UNICO ${ }^{\circledR}$ modelo $2800 \mathrm{H}$ (Dayton, NJ, EUA) y se cuantifica el contenido de compuestos fenólicos mediante una curva estándar.

La curva estándar se obtiene tomando alícuotas de una solución madre de ácido gálico (33 mg de ácido gálico/100mL de agua destilada). Se realizan diluciones de esta solución madre de la siguiente manera ( $\mathrm{mL}$ solución madre de ácido gálico:agua destilada): 0:10, 1:9, 2:8, 3:7, 4:6, 5:5, $6: 4,7: 3,8: 2,9: 1,10: 0$, para obtener 10 puntos de la curva estándar. Para la cuantificación del contenido de compuestos fenólicos se grafican las absorbancias obtenidas (y) y las concentraciones de ácido gálico de las soluciones estándar $(x)$ y se hace una regresión lineal para obtener la ecuación de la curva estándar. Sustituyendo y reacomodando las variables, la ecuación queda de la siguiente manera:

$$
A G=\frac{A-b}{m} * 100
$$

\section{Microbiología}

Se evaluó el conteo de las unidades formadoras de colonias (UFC) en cáscara de tuna para bacterias mesófilas aerobias (BMA) y mohos y levaduras ( $M$ y $L$ ). Primeramente se midió el área superficial promedio de cinco tunas para poder cuantificar las UFC por $\mathrm{cm}^{2}$. Para la medición microbiológica se coloca en una bolsa estéril una tuna con $10 \mathrm{~mL}$ de agua peptonada y se realizó un lavado alrededor de la tuna durante 15 segundos. Posteriormente, se tomó un $\mathrm{mL}$ del agua de lavado para realizar las diluciones necesarias hasta alcanzar el conteo adecuado. Los resultados se informan como UFC/ $\mathrm{cm}^{2}$.

\section{Análisis estadístico}

Los resultados fueron analizados mediante análisis de varianza $(\alpha=0,05)$ utilizando el programa Minitab 14 (Minitab, Inc. PA, EUA). 


\section{RESULTADOS Y DISCUSIÓN}

Caracterización fisicoquímica

En la tabla 1 se muestran los resultados de la caracterización fisicoquímica de la tuna roja San Martín. Los valores publicados por diferentes investigadores para el peso de tuna entera, pulpa y cáscara son diversos y dependen en gran medida de la variedad de tuna. Sin embargo, los resultados obtenidos caen dentro de lo reportado por Moßhammer et al. (2006). Ellos reportaron valores de peso de tuna entera de 67-216 g. El-Samahy et al. (2006) reportó el peso de pulpa y cáscara de tres variedades de tuna Egipcia, con valores de 41,53-49,63 y 39,23-44,53\%, respectivamente. Piga (2004) reportó intervalos de $\mathrm{pH}$, sólidos solubles totales y acidez titulable de $5,3-7,1,10,7-17 \%$ y $0,01-0,18 \%$ (equivalentes de ácido cítrico), respectivamente.

Tabla 1: Características fisicoquímicas de la tuna roja San Martín. La concentración aparece como $\mathrm{X} \pm \sigma$ (media \pm desviación estándar)

\begin{tabular}{cc}
\hline Componente & Concentración $^{\text {a }}$ \\
\hline Peso tuna entera $(\mathrm{g})$ & $99,75 \pm 7,54$ \\
Peso pulpa $(\mathrm{g})$ & $52,2 \pm 4,39$ \\
Peso cáscara $(\mathrm{g})$ & $47,53 \pm 5,32$ \\
$\mathrm{pH}$ & $6,04 \pm 0,01$ \\
Sólidos solubles totales & $14,17 \pm 0,12$ \\
Acidez titulable (ácido cítrico, \%) & $0,036 \pm 0,005$ \\
Índice de Madurez & $393,97 \pm 45,58$ \\
\hline
\end{tabular}

Pérdida de peso

La pérdida de peso (PP) de la tuna se vio afectada de manera significativa $(P<0,05)$ por el tiempo y la temperatura de almacenamiento. La PP después de 28 días de almacenamiento fue de $14,16 \pm 0,6,15,59 \pm 0,38$ y de $9,66 \pm 0,96 \%$, para las temperaturas de $28 \pm 2,9 \pm 2,4 \pm 1^{\circ} \mathrm{C}$, respectivamente. Diferentes investigadores han reportado el efecto del almacenamiento de múltiples variedades de tuna, todos ellos han concluido que la pérdida de peso está en función de las condiciones de almacenamiento, pero sobre todo y en gran medida de la variedad de tuna (Corrales y Hernández, 2005).

La ecuación 7,8 y 9 representan las regresiones lineales de las temperaturas de 28,9 y $4^{\circ} \mathrm{C}$, respectivamente. El coeficiente de correlación es de 0,954 para la temperatura de $9^{\circ} \mathrm{C}$, mientras que para $28^{\circ} \mathrm{C}$ es de 0,970 y de 0,996 para $4^{\circ} \mathrm{C}$.

$\mathrm{PP}=0.48($ tiempo) +1.074

$P P=0.58($ tiempo $)+1.014$

$\mathrm{PP}=0.339($ tiempo $)+0.067$

Se evaluó la pérdida de peso en pulpa y cáscara de tuna. Se observó que el peso de la pulpa de tuna "aumenta" con el tiempo de almacenamiento, mientras que el peso de la cáscara "disminuye"; esto indica que la PP se da principalmente en la cáscara, afectando de manera visible en el grosor de la misma. Corrales y Hernández (2005) concluyeron que esto es debido probablemente a la pérdida de agua en cáscara. Por otro lado, Gonzales et al. (2001) informaron que la pérdida de peso es menor en almacenamiento a $4^{\circ} \mathrm{C}$, comparada con la tuna almacenada a temperatura ambiente. La pérdida de peso y ablandamiento de la cáscara de tuna es muy inconveniente, ya que esto contribuye al incremento en la susceptibilidad al daño físico y deterioro de la tuna durante su manejo (Cantwell et al. 1995). 
Color

En la tabla 2 se presentan los valores de los parámetros $L, a$ y $b$ en pulpa y cáscara de tuna.

Pulpa: El análisis estadístico realizado indica que el valor de $L$, es afectado de manera significativa por la temperatura de almacenamiento $(P<0,05)$. Se observa que a $28^{\circ} \mathrm{C}$ el valor de $L$ disminuye de $32,51 \pm 0,19$ a $24,79 \pm 1,73$, mientras que para las otras temperaturas de almacenamiento no existe diferencia significativa. La disminución de $L$ es probablemente debido a un oscurecimiento en la pulpa de tuna. Mercado et al. (2007) almacenaron diferentes variedades de tuna a $6-8^{\circ} \mathrm{C}$, ellos reportaron que el color rojo de la tuna roja pelona cambia a un color más oscuro debido a que se está disminuyendo el pigmento que absorbe el color azul.

Cáscara: En la tabla 2 se muestran los parámetros de color para cáscara de tuna roja San Martín, se observa que en contra sentido a la pulpa, no se presentó efecto significativo $(P>0,05)$ en el parámetro $L$ con respecto al tiempo y temperatura de almacenamiento. Sin embargo, el valor de $a$ si es afectado por el tiempo de almacenamiento y se observa una disminución en este valor sobre todo a temperatura de $4^{\circ} \mathrm{C}$, mientras que el parámetro $b$ es afectado significativamente $(P<0,05)$ por la temperatura de almacenamiento. Mercado et al. (2007) almacenaron tuna rojo pelón (6$8^{\circ} \mathrm{C}$ ) durante 60 días y observaron que el parámetro $L$ se mantenía durante todo el tiempo de almacenamiento, mientras que de manera general la coloración se tornó más amarillenta después del tiempo en refrigeración, probablemente a la disminución de la concentración en los pigmentos que absorben el color verde.

Tabla 2: Cambio en los parámetros $L, a$ y $b$ en pulpa y cáscara de tuna. Los valores aparecen como $\mathrm{X} \pm \sigma$ (media \pm desviación estándar)

\begin{tabular}{|c|c|c|c|c|c|}
\hline \multirow{3}{*}{\multicolumn{2}{|c|}{ Parámetro Temperatura $\left({ }^{\circ} \mathrm{C}\right)$}} & \multicolumn{2}{|c|}{ Pulpa $^{a}$} & \multicolumn{2}{|c|}{ Cáscara $^{a}$} \\
\hline & & \multicolumn{4}{|c|}{ Tiempo (dia) } \\
\hline & & 0 & 28 & 0 & 28 \\
\hline \multirow[t]{3}{*}{$L$} & 28 & $32,51 \pm 0,19$ & $24,79 \pm 1,73$ & $41,12 \pm 5,83$ & $41,73 \pm 1,62$ \\
\hline & 9 & $32,51 \pm 0,19$ & $32,09 \pm 5,54$ & $41,12 \pm 5,83$ & $34,88 \pm 2,11$ \\
\hline & 4 & $32,51 \pm 0,19$ & $30,62 \pm 1,52$ & $41,12 \pm 5,83$ & $38,83 \pm 1,09$ \\
\hline \multirow[t]{2}{*}{$a$} & $\begin{array}{r}28 \\
9\end{array}$ & $\begin{array}{l}33,59 \pm 0,58 \\
33,59 \pm 0,58\end{array}$ & $\begin{array}{l}33,60 \pm 3,79 \\
33,23 \pm 1,71\end{array}$ & $\begin{array}{l}9,68 \pm 2,50 \\
9,68 \pm 2,50\end{array}$ & $\begin{array}{l}6,47 \pm 1,01 \\
6,87 \pm 3,78\end{array}$ \\
\hline & 4 & $33,59 \pm 0,58$ & $33,30 \pm 4,07$ & $9,68 \pm 2,50$ & $2,34 \pm 1,12$ \\
\hline \multirow[t]{3}{*}{$b$} & 28 & $12,63 \pm 1,60$ & $11,48 \pm 0,90$ & $6,97 \pm 0,98$ & $6,48 \pm 2,26$ \\
\hline & 9 & $12,63 \pm 1,60$ & $11,70 \pm 2,19$ & $6.97 \pm 0,98$ & $8,48 \pm 1,88$ \\
\hline & 4 & $12,63 \pm 1,60$ & $13,52 \pm 0,67$ & $6,97 \pm 0,98$ & $8,74 \pm 1,43$ \\
\hline
\end{tabular}

Resistencia a la penetración

Pulpa: La resistencia a la penetración en pulpa de tuna es afectada por la temperatura de almacenamiento, cambiando de una resistencia inicial de $1,73 \pm 0,6 \mathrm{~N}$ a $0,78 \pm 0,27,0,97 \pm 0,44$ y $1,76 \pm 0,63 \mathrm{~N}$, para las temperaturas de 28,9 y $4^{\circ} \mathrm{C}$, respectivamente, después de 28 días de almacenamiento. Todas las frutas y hortalizas sufren cambios durante su maduración y almacenamiento. Estos son debido a transformaciones bioquímicas, que provocan cambios en la textura, ocasionando la pérdida de la firmeza (Aguilar et al. 2007). Sin embargo, existe una relación directamente proporcional entre la temperatura y la actividad metabólica del fruto, que evita que este se ablande a temperaturas de refrigeración (Mercado et al. 2007; Guerra y Casquero, 2005). Estadísticamente ni la temperatura ni el tiempo de almacenamiento afectan de manera significativa $(P>0,05)$.

Cáscara: La cáscara de tuna presentó un comportamiento diferente ya que solo la temperatura afectó significativamente $(P<0,05)$ la resistencia a la penetración. Esto pudo deberse a que a 
temperatura ambiente la humedad relativa fue más baja comparada con las otras temperaturas de almacenamiento; esto pudo provocar mayor pérdida de humedad y por lo tanto que la cáscara se hiciera menos flexible. Como consecuencia se aceleran los procesos degradativos generando que la cáscara pueda ser penetrada con mayor facilidad (Cantwell et al. 1995). Mercado et al. (2008) evaluaron el esfuerzo de penetración en cáscara y pulpa de tuna y observaron que las propiedades relacionadas con la resistencia de los tejidos que conforman los frutos tienden a disminuir durante el tiempo de almacenamiento. Por otro lado, Corrales y Hernández (2005) indican que la resistencia a la penetración en cáscara de tuna está en función de la variedad de ésta.

\section{Pectinesterasa (PE)}

En la tabla 3 se presentan los valores de PE. Se observa que existe una disminución en la PE a las tres temperaturas de almacenamiento durante los primeros 21 días. Posterior a este tiempo no se observan cambios significativos $(P>0,05)$ en la actividad enzimática. Diferentes autores han informado que la PG actúa después de que la PE hidroliza los enlaces éster de los grupos metilo de las cadenas poligalacturonasas (Lefever et al. 2004; Giovannoni, 2001). Puede ser que el ablandamiento de la tuna se deba a la actividad de la enzima PG. Gurrieri et al. (2000) observaron bajos valores de actividad de PE en jugo de tuna, comparado con otras frutas, y lo atribuyeron a que la enzima puede estar presente en cantidades muy pequeñas o bien que no es muy eficiente en la degradación de la pectina. Carrillo et al. (2002) analizaron la actividad de diferentes enzimas hidrolíticas en variedades de tuna naranjona y charola. Ellos reportaron que la actividad de la PE en tuna naranjona aumentó durante los primeros seis días de almacenamiento y posteriormente se mantuvo constante. Mientras que en tuna charola, la actividad enzimática se mantuvo sin variación durante 48 días de almacenamiento a $18^{\circ} \mathrm{C}$. El análisis de varianza muestra que el tiempo de almacenamiento es un factor significativo $(P<0,05)$ para la actividad de la $P E$, mientras que la temperatura de almacenamiento no afecta $(P>0,05)$ la actividad de la $P E$.

Tabla 3: Actividad de la enzima pectinesterasa ${ }^{\psi}$ y polifenoloxidasa ${ }^{\ddagger}$ en pulpa y cáscara de tuna. Los valores aparecen como $\mathrm{X} \pm \sigma$ (media \pm desviación estándar)

\begin{tabular}{|c|c|c|c|c|c|}
\hline \multirow[b]{2}{*}{ Enzima } & \multirow[b]{2}{*}{ Temperatura $\left({ }^{\circ} \mathrm{C}\right)$} & \multicolumn{4}{|c|}{ Tiempo (día) ${ }^{\mathrm{a}}$} \\
\hline & & 0 & 28 & 0 & 28 \\
\hline $\begin{array}{l}\mathrm{UAE}^{\Psi} / \mathrm{mL} \\
\text { de pulpa }\end{array}$ & $\begin{array}{r}28 \\
9 \\
4\end{array}$ & $\begin{array}{l}1,9 \mathrm{E}-3 \pm 1,4 \mathrm{E}-4 \\
1,9 \mathrm{E}-3 \pm 1,4 \mathrm{E}-4 \\
1,9 \mathrm{E}-3 \pm 1,4 \mathrm{E}-4\end{array}$ & $\begin{array}{l}1,6 \mathrm{E}-3 \pm 3,5 \mathrm{E}-5 \\
1,5 \mathrm{E}-3 \pm 2,4 \mathrm{E}-4 \\
1,6 \mathrm{E}-3 \pm 1,4 \mathrm{E}-4\end{array}$ & $\begin{array}{l}1,5 \mathrm{E}-3 \pm 1,5 \mathrm{E}-5 \\
1,5 \mathrm{E}-3 \pm 9,1 \mathrm{E}-6 \\
1,4 \mathrm{E}-3 \pm 1,1 \mathrm{E}-4\end{array}$ & $\begin{array}{l}1,5 \mathrm{E}-3 \pm 1,4 \mathrm{E}-5 \\
1,5 \mathrm{E}-3 \pm 1,7 \mathrm{E}-5 \\
1,5 \mathrm{E}-3 \pm 7,0 \mathrm{E}-6\end{array}$ \\
\hline $\begin{array}{l}\text { UAE } ¥ / g \\
\text { de pulpa }\end{array}$ & $\begin{array}{r}28 \\
9 \\
4\end{array}$ & $\begin{array}{l}546 \pm 144 \\
546 \pm 144 \\
546 \pm 144\end{array}$ & $\begin{array}{l}1800 \pm 170 \\
2080 \pm 509 \\
2160 \pm 679\end{array}$ & $\begin{array}{c}2868 \pm 390 \\
4080 \pm 51 \\
3456 \pm 0\end{array}$ & $\begin{array}{c}936 \pm 170 \\
102 \pm 42 \\
132 \pm 17\end{array}$ \\
\hline $\begin{array}{l}\text { UAE } ¥ / g \\
\text { de cáscara }\end{array}$ & $\begin{array}{r}28 \\
9 \\
4\end{array}$ & $\begin{array}{l}59,25 \pm 31 \\
59,25 \pm 31 \\
59,25 \pm 31\end{array}$ & $\begin{array}{c}1200 \pm 0 \\
330 \pm 195 \\
60 \pm 17\end{array}$ & $\begin{array}{c}1680 \pm 0 \\
840 \pm 167 \\
480 \pm 0\end{array}$ & $\begin{array}{c}1428 \pm 17 \\
540 \pm 51 \\
48 \pm 0\end{array}$ \\
\hline
\end{tabular}

\section{Polifenoloxidasa}

En la tabla 3 se muestran los datos de la actividad de la polifenoloxidasa en pulpa y cáscara de tuna.

Pulpa. El tiempo de almacenamiento es el factor que afecta de manera significativa $(P<0,05)$ la actividad de la PPO. Sin embargo, se observa que a mayor temperatura la actividad de la PPO es menor. Vela et al. (2001) observaron que la actividad de la polifenoloxidasa en mango fue mayor a temperaturas de refrigeración $\left(6^{\circ} \mathrm{C}\right)$, con respecto a temperaturas de almacenamiento de $25^{\circ} \mathrm{C}$. Lo anterior puede deberse a que durante el almacenamiento en refrigeración se produce daño por frío al interior del fruto con la consecuente liberación de metabolitos tales como aminoácidos, azúcares, minerales y compuestos fenólicos, así como liberación de compuestos de la pared 
celular, que al entrar en contacto con la enzima producen melanoidinas (Vela et al. 2001). No obstante, Balois et al. (2007) informan que la disminución de la temperatura es un factor inhibitorio de la actividad de la polifenoloxidasa, demostrando que en pitahayas almacenadas a $3^{\circ} \mathrm{C}$, la actividad fue significativamente menor que en aquellas almacenadas a 7,11 y $22^{\circ} \mathrm{C}$. Por otra parte, Baquero et al. (2005) reportaron que la actividad enzimática era consecuencia de la senescencia del fruto, que desencadenaba la activación de formas latentes, solubilización de las enzimas unidas a la pared celular y a membranas de organelos o bien por síntesis de novo.

Cáscara. La cáscara de la tuna presentó comportamientos inversos a los presentados en la pulpa, ya que a temperatura de $28^{\circ} \mathrm{C}$ la actividad de la enzima fue mayor significativamente $(P<0,05)$ comparada con las otras dos temperaturas de almacenamiento. Durante el almacenamiento se observo que no existía oscurecimiento enzimático en toda la tuna. A bajas temperaturas se pigmentaba con pequeños puntos color café, mientras que a temperatura de $28^{\circ} \mathrm{C}$ la parte media de la tuna se oscurecía con manchas. La pigmentación en cáscara a bajas temperaturas puede deberse al daño por frío explicado anteriormente.

\section{Actividad antioxidante}

En la tabla 4 se presentan los valores de actividad antioxidante en pulpa de tuna roja San Martín. Se observa una tendencia a aumentar con el paso del tiempo. Esto es corroborado con el análisis estadístico, el cual mostró que el tiempo de almacenamiento tiene efectos significativo sobre la actividad antioxidante $(P<0,05)$, no así la temperatura de almacenamiento. De la misma manera que el contenido de compuestos fenólicos la actividad antioxidante está en función de la variedad de tuna y del contenido de pigmentos. Los valores de las distintas variedades se encuentran desde 2,24 $\pm 0,09$ hasta 5,31 $\pm 0,49 \mu \mathrm{mol}$ equivalentes de Trolox/g en pulpa (Stintzing et al. 2005; Carrasco y Encina, 2008). Los valores obtenidos en este estudio se encuentran alrededor de 0,76$0,94 \mu \mathrm{mol}$ equivalentes de Trolox/g de jugo de tuna; valores que están por debajo de los observados por Stintzing et al. (2005). Carrasco y Encina (2008) informan que la capacidad antioxidante de tuna roja medida mediante el método de DPPH fue de $77,65 \%$, que es mayor que en tunas de otras coloraciones. Por otro lado, Galati et al. (2003) indicaron que solo la fracción acuosa tiene actividad antioxidante, no así la parte orgánica. Aún más importante fue el demostrar que el jugo de tuna tenía efectos benéficos en la prevención de ulceras estomacales en ratas tratadas con etanol.

Tabla 4: Actividad antioxidante y compuestos fenólicos de pulpa de tuna roja San Martín. Los valores aparecen como $\mathrm{X} \pm \sigma$ (media \pm desviación estándar).

\begin{tabular}{ccccccc}
\hline & \multicolumn{3}{c}{$\begin{array}{c}\text { Actividad antioxidante }(\mathrm{mg} \text { Trolox/) } \\
100 \mathrm{~mL} \text { de jugo }\end{array}$} & \multicolumn{3}{c}{$\begin{array}{c}\text { Compuestos fenólicos (mg ac. gálicol } \\
100 \mathrm{~mL} \text { de jugo })^{\mathrm{a}}\end{array}$} \\
\hline Tiempo (dias) & $28^{\circ} \mathrm{C}$ & $9^{\circ} \mathrm{C}$ & $4^{\circ} \mathrm{C}$ & $28^{\circ} \mathrm{C}$ & $9^{\circ} \mathrm{C}$ & $4^{\circ} \mathrm{C}$ \\
\hline 0 & $12,27 \pm 0,92$ & $12,27 \pm 0,92$ & $12,27 \pm 0,92$ & $42,01 \pm 6,08$ & $42,01 \pm 6,08$ & $42,01 \pm 6,08$ \\
7 & $12,91 \pm 0,49$ & $12,44 \pm 2,37$ & $12,31 \pm 3,22$ & $42,73 \pm 1,74$ & $49,62 \pm 0,95$ & $35,65 \pm 4,91$ \\
14 & $21,79 \pm 0,39$ & $20,77 \pm 0,04$ & $21,93 \pm 0,34$ & $48,27 \pm 3,09$ & $43,76 \pm 0,29$ & $41,25 \pm 1,15$ \\
21 & $21,48 \pm 0,37$ & $23,67 \pm 0,10$ & $22,21 \pm 0,90$ & $42,61 \pm 3,15$ & $39,95 \pm 3,20$ & $50,04 \pm 1,18$ \\
\hline
\end{tabular}

Compuestos fenólicos

En la tabla 4 se presenta el contenido de compuestos fenólicos en pulpa de tuna. A 28 y $9^{\circ} \mathrm{C}$ se observa que estos compuestos se mantienen durante el tiempo de almacenamiento, mientras que a $4^{\circ} \mathrm{C}$ se presenta una tendencia a aumentar. Sin embargo, ni el tiempo ni la temperatura de almacenamiento tienen efectos significativos $(P>0,05)$ sobre el contenido de compuestos fenólicos. Los principales compuestos fenólicos en la tuna son la isorhamnetin-3-rutinosido y pequeñas cantidades de isorhamnetin-3-glucosido, rutin y kaempferol-3-rutinosido (Galati et al. 2003; Stintzing et al. 2001). Galati et al. (2003) observaron valores de 74,6 mg de ácido gálico/100 $\mathrm{mL}$ de jugo de tuna entera variedad siciliana. Por otro lado, Stintzing et al. (2005) obtuvieron valores de $24,2 \pm 13,4,24,7 \pm 23,1,33,5 \pm 19,3,66 \pm 35,8 \mathrm{mg}$ ácido gálico/100 $\mathrm{mL}$ de jugo de tuna verde, naranja, roja y morada, respectivamente. Stintzing et al. (2005) y Carrasco y Encina (2008) 
concluyeron que el contenido de compuestos fenólicos está en función de la variedad de tuna y que estos son los principales responsables de la actividad antioxidante. Piga et al. (2003) informaron que el contenido de compuestos fenólicos disminuía en tuna mínimamente procesada almacenada en refrigeración y era probablemente debido a que tenía un efecto protector sobre la actividad antioxidante y el ácido ascórbico.

\section{Carga microbiana}

En la Figura 1 se presentan las curvas de crecimiento microbiano (bacterias mesófilas aerobias (BMA) y mohos y levaduras ( $M$ y $L)$ ) sobre la superficie de tuna. Después de 28 días de almacenamiento se observó que el recuento de BMA fue de 2366, 227 y $591 \mathrm{UFC/ \textrm {cm } ^ { 2 }}$ para las temperaturas de almacenamiento de 28,9 y $4^{\circ} \mathrm{C}$, respectivamente. Mientras que para $\mathrm{M}$ y $\mathrm{L}$ el recuento fue de 1047,173 y $319 \mathrm{UFC} / \mathrm{cm}^{2}$, para 28,9 y $4^{\circ} \mathrm{C}$, respectivamente. Hasta ahora existen pocos estudios que informan la actividad microbiana en tuna entera. Sin embargo, como en todas las frutas y hortalizas, las tunas deben refrigerarse para alargar su vida útil, ya que esto frena el crecimiento microbiano, así como los procesos químicos y bioquímicos (Aguayo, 2003). Gonzales et al. (2001) almacenaron tuna variedad burrona a 28 y $4^{\circ} \mathrm{C}$. Ellos informaron que la presencia de mohos se observó hasta el día 60 de almacenamiento a $4^{\circ} \mathrm{C}$, mientras que a temperatura ambiente la descomposición se presento al día 45, estos resultados concuerdan con lo obtenido en este estudio. El análisis estadístico mostró que el tiempo y la temperatura de almacenamiento tuvieron un efecto significativo $(P<0,05)$ sobre el crecimiento microbiano.

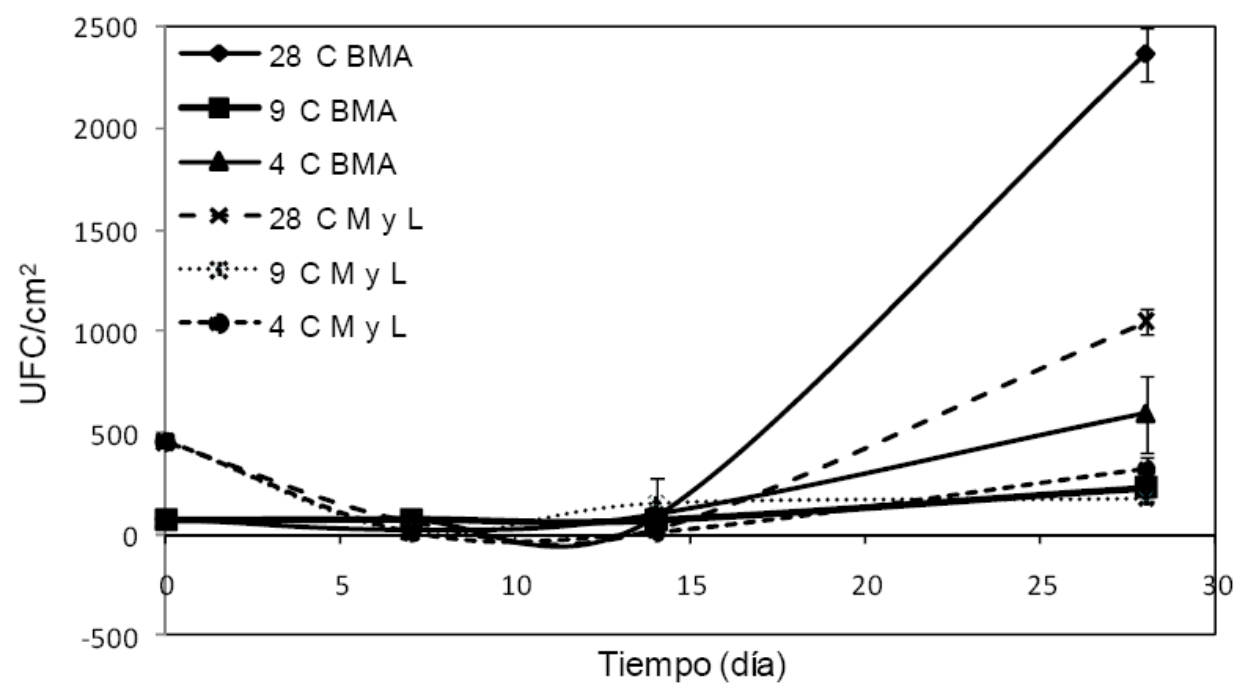

Fig. 1: Cuenta microbiana de tuna roja San Martín almacenada a diferentes temperaturas.

\section{CONCLUSIONES}

Las bajas temperaturas de almacenamiento ayudan a aumentar la vida útil de la tuna roja San Martín, disminuyendo la pérdida de peso, resistencia a la penetración, el oscurecimiento enzimático y la actividad microbiana. Sin embargo, no tienen efecto sobre la pectinesterasa y los compuestos antioxidantes. Por otra parte, el tiempo de almacenamiento es el factor más determinante en la calidad de la tuna. Estos estudios podrían ayudar a los productores de tuna del Estado de Puebla al seleccionar la temperatura de almacenamiento de la tuna roja San Martín, ya que la tuna se mantuvo con buen aspecto físico hasta los 28 días de almacenamiento.

\section{NOTACIÓN}

$\begin{array}{ll}A_{f} & \text { Absorbancia final } \\ A G & \text { Trolox }(\mathrm{mg} / \mathrm{mL}) \\ A_{i} & \text { Absorbancia inicial }\end{array}$




$\begin{array}{ll}b & \text { Intercepto } \\ g & \text { Peso o volumen usado para hacer el extracto enzimático }(\mathrm{g} \circ \mathrm{mL}) \\ m & \text { Pendiente de la recta }(\mathrm{mL} \text { de } \mathrm{NaOH} / \text { tiempo }) \\ m & \text { Pendiente de la recta (absorbancia/tiempo) } \\ m & \text { Pendiente de la recta }(\% / \mathrm{mg} / \mathrm{mL}) \\ N_{N a O H} & \text { Concentración de } \mathrm{NaOH} \\ U A E & \text { Unidad de actividad enzimática } \\ U A E & \text { Unidad de actividad enzimática } \\ U I & \text { Inhibición }(\%) \\ U I & \text { Inhibición }(\%) \\ V & \text { Volumen total de muestra homogenizada con buffer }(\mathrm{mL}) \\ V & \text { Volumen de extracto enzimático usado para la reacción }(\mathrm{mL}) \\ V_{j u g o} & \text { Volumen de jugo }(\mathrm{mL})\end{array}$

\section{AGRADECIMIENTOS}

Los autores agradecen al Consejo Nacional de Ciencia y Tecnologia (CONACyT) por el financiamiento recibido en esta investigación.

\section{REFERENCIAS}

Aguayo, E, Innovaciones tecnológicas en la conservación de melón y tomate procesado en fresco, Tesis de doctorado, Universidad Politécnica de Cartagena, España (2003).

Aguilar, L, M. T., Martínez, A., Barrientos, N., Aguilar y C. Gallegos, Potencial de oscurecimiento enzimático de variedades de nopalitos. Journal of the Professional Association for Cactus Development: 9,122-135 (2007).

AOAC, Official Methods of Analysis. $14^{a}$ edición. Association of Official Analytical Chemists, Inc. Washington D.C., EUA (2000).

Balois, R., M.T. Colinas, C.B. Peña, S.H. Chávez-Franco y L. Alia, Sistema de estrés oxidativo, fenoles-polifenol oxidasa-peroxidasa, de frutos de pitahaya (Hilocerus undatus) almacenados con frío. Revista Chapingo, Serie horticultura: 13(2), 115-120 (2007).

Baquero, L., J. Castro y C. Narváez, Catalasa, peroxidasa y polifenoloxidasa en pitaya amarilla (Acanthocereus pitajaya): Maduración y senescencia, Acta Biológica Colombiana: 10(2), 49-59 (2005).

Cantwell, M., Postharvest management of fruits and vegetables stems. En Agro-ecology, Cultivation and Uses of Cactus Pear, G. Barbera, P. Inglese and E. Pimienta-Barrios (eds.), FAO Plant Production and Protection Paper 132, pp.120-143 Roma, Italia (1995).

Carrasco, R. y C. Encina, Determinación de la capacidad antioxidante y compuestos bioactivos de frutas nativas peruanas, Revista de la Sociedad Química del Perú: 74(2), 108-124 (2008).

Carrillo, A., A. Cruz, A. Cárabez, F. Guevara y O. Paredes, Hydrolytic activity and ultrastructural changes in fruit skins from two prickly pear (Opuntia spp.) varieties during storage, Journal of Agricultural and Food Chemistry: 50, 1681-1685 (2002). 
Corrales, J. y J. Hernández, Cambios en la calidad postcosecha de variedades de tuna con y $\sin$ semilla, Revista Fitotecnia Mexicana: 28, 9-16 (2005).

Del Valle, V., P. Hernández, A. Guarde y M. Galotto, Development of a cactus mucilage edible coating (Opuntia ficus-indica) and its application to extend strawberry (Fragaria ananassa) shelflife, Food Chemistry: 91, 751-756 (2005).

El-Samahy, S.K., A.A. El-Hady, A. Habiba y T.E. Moussa, Chemical and rheological characteristics of orange-yellow cactus-pear pulp from Egypt, Journal of the Professional Association for Cactus Development: 8, 39-51 (2006).

Esquivel, P., Los frutos de las cactáceas y su potencial como materia prima, Agronomía Mesoamericana: 15(2), 215-219 (2004).

Galati, E.M., M.R. Mondello, D. Giuffrida, G. Dugo, N. Miceli, S. Pergolizzi y M. F. Taviano, Chemical characterization and biological effects of Sicilian Opuntia ficus-indica (L.) Mill. Fruit juice: antioxidant and antiulcerogenic activity, Journal of Agricultural and Food Chemistry: 51, 4903-4908 (2003).

Gao, X., M. Ohlander, N. Jeppsson, L. Bjork y V. Traljkovski, Changes in antioxidant effects and their relationship to phytonutrients in fruits of sea L. during maturation Buckthorn (Hippophae rhamnoides), Journal of Agricultural and Food Chemistry: 48, 1485-1490 (2000).

Guerra, M. y P.A. Casquero, Evaluación de la madurez de manzana y pera en almacenamiento frigorífico conjunto con absorvedor de etileno, Información Tecnológica: 16(4), 11-16 (2005).

Giovannoni, J., Molecular biology of fruit maturation and ripening. Annu. Review of Plant Physiology and Plant Molecular Biology: 52, 725-749 (2001).

Gonzales, R., T. Morales, E. Olivares, J. Aranda y C. Gallegos, Conservación de una variedad de tuna (burrona) bajo diferentes manejos poscosecha, Ciencia, Universidad Autónoma de Nuevo León, México: 4, 322-329 (2001).

Gurrieri, S., L. Miceli, C.M. Lanza, F. Tomaselli, R.P. Bonomo y E. Rizzarelli, Chemical characterization of Sicilian prickly pear (Opuntia ficus-indica) and perspectives for the storage of its juice, Journal of Agricultural and Food Chemistry: 48, 5424-5431 (2000).

Kader, A.A., D. Zagory y E.L. Kerbel, Modified atmosphere packaging of fruits and vegetables, Critical Reviews in Food Science and Nutrition: 28(1), 1-30 (1989).

Kuskoski, M., A. Asuero, M. Parrilla, A. Troncoso y R. Fett, Actividad antioxidante de pigmentos antociánicos, Ciencia y Tecnología de Alimentos (Campinas): 24, 691-693 (2004).

Lefever, G., M. Vieuille, N. Delage, A. D’Harlingue, J. De Monteclerc y G. Bompleix, Characterization of cell wall enzyme activities, pectin composition, and technological criteria of strawberry cultivars (Fragaria $\times$ ananassa Duch), Food Chemistry and Toxicology: 69(4), 221-226 (2004).

Mercado, J., M. López, G. Martínez y M. Arias, Estudio de las propiedades reológicas de la tuna en fresco. IX Congreso de Ciencia de los Alimentos y V Foro de Ciencia y Tecnología de Alimentos. Guanajuato, Gto., México. Mayo 31 (2008).

Mercado, J., M. López, G. Martínez, J. Sarahí y S. Arévalo, Estudio de las propiedades fisicoquímicas de las variedades de tuna rojo pelón (Opuntia ficus-indica), morada, reina (Opuntia amyclaea) y Xoconostle (Opuntia matudae scheinvar) bajo almacenamiento refrigerado, IX Congreso de Ciencia de los Alimentos y $\mathrm{V}$ foro de Ciencia y Tecnología de Alimentos, Guanajuato, Gto., México, Mayo 31 (2007). 
Moßhammer, M.R., F.C. Stintzing y R. Carle, Cactus Pear Fruits (Opuntia spp.): A Review of Processing Technologies and Current Uses, Journal of the Professional Association for Cactus Development: 8, 1-25 (2006)

Ochoa, C.E. y J.A. Guerrero, La tuna: una perspectiva de su producción, propiedades y métodos de conservación, Temas Selectos de Ingeniería de Alimentos, Universidad de las Américas Puebla, México: 4(1), 49-63 (2010).

Piga, A., A. Del Caro, I. Pinna y M. Agabbio, Changes in ascorbic acid, polyphenol content and antioxidant activity in minimally processed cactus pear fruits, Lebensmittel-Wissenschaft undTechnologie: 36, 257-262 (2003).

Piga, A., Cactus pear: A fruit of nutraceutical and functional importance, Journal of the Professional Association for Cactus Development: 6, 9-22 (2004).

Pizzocaro, F., D. Torreggiani y G. Gilardi, Inhibition of Apple polyphenoloxidase (PPO) by ascorbic acid, citric acid and sodium chloride, Journal of Food Processing and Preservation: 17, 21-30 (1993).

Re, R., N. Pellegrini, A. Proteggente, A. Pannala, M. Yang y C. Rice-Evans, Antioxidant activity applying an improved ABTS radical catión decolorization assay, Free Radical Biology and Medicine: 26, 1231-1237 (1999).

Rouse, A.H., C.D. Atkins y R.L. Huggart, Effect of pulp quantity on chemical and physical properties of citrus juices and concentrates, Food Technology: 8, 431-435 (1954).

Stintzing, F.C., A. Schieber y R. Carle, Phytochemical and nutritional significance of cactus pear, European Food Research and Technology: 212, 396-407 (2001).

Stintzing, F.C., K.M. Herbach, M.R. Mosshammer, R. Carle, W. Yi, S. Sellappan, C.C. Akoh, R. Bunch y P. Felker, Color, betalain pattern, and antioxidant properties of cactus pear (Opuntia spp.) clones, Journal of Agricultural and Food Chemistry: 53, 442-451 (2005).

Tesoriere, L., D. Butera, M. Allegra, M. Fazzari y M.A. Livrea, Distribution of betalain pigments in red blood cells after consumption of cactus pear fruits and increased resistance of the cells to ex vivo induced oxidative hemolysis in humans, Journal of Agricultural and Food Chemistry: 53, 12661270 (2005).

Vela, G., D. León, H.S. García y J. De la Cruz, Estrés por frío y actividad de la polifenoloxidasa en mango (Mangifera indica cv. Manila). En IX Congreso Nacional de Biotecnología y Bioingeniería. XIII Congreso Nacional de Ingeniería Bioquímica. II Congreso Internacional de Ingeniería Bioquímica. Veracruz, Veracruz, México, 10-14 de septiembre (2001). 